\title{
LA POLITICA PRESUPUESTARIA DE LA DICTADURA \\ DE PRIMO DE RIVERA: \\ ¿UNA RECONSIDERACION NECESARIA? *
}

JORDI PALAFOX GAMIR

Universidad de Valencia

La política económica desarrollada por la dictadura del general Primo de Rivera ha sido objeto, durante los últimos años, de una atención considerable. Desde que el profesor Velarde publicara su pionera aportación ', numerosos especialistas han abordado, desde distintas perspectivas y con una valoración también divergente, el estudio de las principales medidas económicas adoptadas por el régimen autoritario durante los siete años en que estuvo en el poder. En todas ellas, sin embargo, se ha tendido a reconocer una importancia notable a la gestión de las finanzas públicas. Bien para destacar su relevancia en el crecimiento de la renta, bien para criticar el marasmo financiero que Calvo Sotelo provocó, la política presupuestaria ha constituido uno de los elementos clave en la valoración del período que transcurre entre septiembre de 1923 y enero de 1930 , y más concretamente entre diciembre de 1925 y el 20 de enero de 1930, etapa en lla que Calvo Sotelo estuvo al frente del Ministerio de Hacienda.

Recientemente, en un sugestivo y provocador artículo, Pablo Martín Aceña y Francisco Comín ${ }^{2}$ han planteado con contundencia la necesidad de proceder a una reinterpretación de la que ellos denominan la visión tradicional de la política monetaria y fiscal del período de entreguerras. Entre otros aspectos, su aportación ha puesto de manifiesto las insuficiencias de los análisis previos sobre el papel desempeñado por el Estado en el fomento de la actividad y la necesidad de considerar con mayor rigor las relaciones entre ambos conjuntos de medidas a la hora de valorar las decisiones adoptadas por las autoridades económicas entre 1923 y $1936^{3}$.

* Una primera versión de este artículo fue presentada al Curso sobre La Hacienda Pública durante la dictadura de Primo de Rivera, que, organizado por la Universidad Internacional Menéndez y Pelayo y el Instituto de Estudios Fiscales, tuvo lugar en Santander durante el verano de 1985 . Quiero agradecer a Leticia Alvarez, Teresa Carnero, Francisco Comín, Josep Fontana, Pablo Martín Aceña y Gabriel Tortella las críticas y comentarios que han hecho a los borradores previos. Como es usual, las páginas que siguen son exclusivamente responsabilidad de su autor.

J. Velarde (1968).

2 F. Comín Comín y P. Martín Aceña (1984).

${ }^{3}$ Lo que constituye, sin duda, uno de los méritos más evidentes del libro de Pablo Martín Aceña. P. Martín Aceña (1984). 
El objetivo de este artículo es plantear una interpretación alternativa a la que puede deducirse de sus conclusiones en relación con la política presupuestaria de la Dictadura. En buena medida, sin embargo, la tesis que aquí se pretende defender y la expuesta por Comín y Martín Aceña no son contrarias, sino complementarias. Su énfasis en los moderados efectos macroeconómicos de la política de gasto no es, al menos así intento demostrarlo a continuación, incompatible con que ésta tuviera una notable importancia en la positiva evolución de sectores concretos que encontraron en el gasto público un mecanismo de expansión o mantenimiento de la demanda que aplazó la imprescindible reducción de sus costos medios de producción. Lo que se intenta subrayar en estas páginas, dentro de un marco más general que considera otros aspectos de la política presupuestaria, es esta cuestión y no la primera.

Deseo insistir, además, en que las páginas que siguen no pretenden ser una alternativa global a la planteada por ellos para la etapa 1923-1935, sino, más modestamente, ofrecer una interpretación diferente de la función desempeñada por el gasto público entre 1923 y 1930.

Ello no quiere decir que las diferencias, en el enfoque o en las conclusiones, sean escasas. Entre las primeras, la más destacada es, quizá, el que en este artículo se analiza el gasto público durante la Dictadura en relación con una visión, que tal vez es la que ellos denominan tradicional, de la evolución de la economía entre 1891 y 1936 en la cual el régimen de Primo de Rivera es considerado como una etapa de auge de los mecanismos de «asistencia» al desarrollo no competitivo del capitalismo en España. Desde el punto de vista de las conclusiones, la principal divergencia se refiere, como se verá, a la cuantía del déficit que debe de ser imputado a la gestión del principal ministro de Hacienda del dictador y a su función. Y me interesa subrayar desde el principio que no es objetivo del artículo el determinar el volumen exacto del mismo, sino mostrar, aun a riesgo de que el enfoque pueda ser criticado de contablemente impreciso, que la política presupuestaria tuvo unos grandes beneficiarios, los empresarios de la gran industria ${ }^{4}$, y un coste de oportunidad no menor: no abordar, en contra de las declaraciones de Calvo Sotelo y Primo de Rivera, la resolución de los notables problemas de la Hacienda ni los no menos graves que tenía planteados la estructura industrial española ${ }^{3}$.

4 Siderurgia, construcción de maquinaria, ferrocarriles y minería del carbón, fundamentalmente.

${ }_{5}^{5}$ No es ésta, pues, una aportación al intenso debate oral entre Francisco Comín y Angel Melguizo, los dos mejores especialistas de la Hacienda durante esta etapa, acerca de qué fuentes son las más precisas para llegar a determinar la cuantía exacta del déficit. Por razones de operatividad, se sigue en estas páginas el criterio adoptado por Francisco Comín y Pablo Martín Aceña en el artículo mencionado. 
Teniendo en cuenta el punto de partida que acabo de resumir, antes de entrar en el aspecto central del análisis me parece imprescindible esbozar algunos rasgos del contexto económico y social anterior a 1923; un contexto que es decisivo para valorar cuáles fueron sus objetivos fundamentales y cuáles, sobre todo, sus logros y sus limitaciones. El punto de partida no puede ser otro que el lento crecimiento de la economía española en los decenios anteriores ante la debilidad de la demanda interna, pero, también, ante la incapacidad de la oferta para aprovechar la elasticidad-precio superior a la unidad que, sin ninguna duda, existía en los principales sectores. Por más que sean discutibles, y «quede un amplísimo terreno de incertidumbre en el conocimiento de la evolución de la renta nacional española, tanto para el siglo XIX como para el $x{ }^{6}{ }^{6}$, parece claro que las diferentes estimaciones recientes de la renta per capita española ${ }^{7}$ desde 1890 hasta 1910 muestran, a pesar del modesto nivel inicial, un aumento claramente insuficiente para acortar la distancia que separaba a la economía española de la de los principales países europeos.

Esta situación puede ser considerada el resultado de lo limitado de las transformaciones de la estructura productiva durante los decenios anteriores, en la que, junto a un sector agrario incapaz en su inmensa mayoría de adaptarse al proceso de transformación que le permitiera competir frente a los productos extranjeros, el sector secundario tendió más a adaptarse al bajo ritmo de expansión de la demanda interna, consecuencia del hecho anterior, que a impulsar un proceso de transformación de las interrelaciones sectoriales básicas que diera como resultado a medio y largo plazo un crecimiento sostenido y sin estrangulamientos.

Se ha insistido en diversas ocasiones en la responsabilidad del sector agrario en este proceso ${ }^{8}$. Pero debe subrayarse que los principales sectores industriales (textil y siderurgia) mantuvieron en todo momento un comportamiento económico de adaptación pasiva ${ }^{9}$ frente al marco determinado por el atraso del sector agrario ${ }^{10}$.

- A. Carreras (1985), p. 20.

7 Además de la de Carreras (1985), que es la que arroja un resultado más positivo, P. Bairoch (1976), N. F. R. Crafts (1983) y L. Prados de la Escosura (1982) y (1984).

Tesis que, sin duda, entronca con la argumentación desarrollada por Nadal en su influyente J. Nadal (1975). Aun cuando hoy, en gran medida gracias a los trabajos del Grupo de Estudios de Historia Rural, la tesis del estancamiento agrario no puede ser defendida - un excelente resumen en R. Garrabou y J. Sanz (1985)-, es evidente que entre 1830 y 1930 el crecimiento agrario fue muy modesto. G. Tortella (1985), pp. 82-86.

- La expresión, bastante feliz para describir el comportamiento del empresariado español a lo largo de su historia, es de J. V. Sevilla (1985).

10 P. Fraile (1985 a) y (1985 b). Para principios de siglo y en el sector siderúrgico, véase M. González Portilla (1981) y (1985). 
Una situación como la anterior, dominada por la falta de innovación y el recurso a la protección arancelaria, no podía dejar de tener su correspondencia en la estructura de los Presupuestos del Estado. La permanente insuficiencia de los ingresos, ante la negativa rotunda a impulsar un sistema fiscal no ya progresivo, sino simplemente proporcional, y las necesidades crecientes de prestar los mínimos servicios por parte del sector público, llevaron a un déficit crónico de la Hacienda. Un desequilibrio entre gastos e ingresos imposible de anular en la práctica por la negativa a contribuir de unos y las necesidades de una sociedad que exigía un sector público más amplio y eficiente ". La importancia creciente de los ingresos por Aduanas sobre las importaciones totales $(11,22$ por 100 en $1920-1922,23,01$ por 100 en 19281930 y cerca de la cuarta parte de los derechos reconocidos y liquidados -excluidos los recursos de negociación de la deuda- durante los mismos años) permite plantear la validez del argumento del «gobierno naciente» a la política arancelaria española de la tercera década del siglo $\mathrm{xx}^{12}$.

Sobre esta situación se superpuso el impacto brutal, tanto en el terreno económico como en el social, de la I Guerra Mundial ${ }^{13}$. Una coyuntura marcada por la especulación, durante la cual la inversión productiva no parece haber seguido la trayectoria de los beneficios ${ }^{14}$, que llevó a un sensible aumento de la tensión social y aceleró el desgaste del sistema político de la Restauración, incapaz de integrar plenamente a las nuevas fuerzas sociales que la modesta, pero evidente, modernización del país había generado. La erosión que provocaron estos dos hechos en la débil legitimación política del Estado restauracionista le llevó a una crisis que, ante la ausencia de alternativas por parte de los partidos tradicionales y la radicalización del movimiento obrero catalán, puede explicar en buena medida el golpe de Estado de 1923 y la escasa reacción social ante la suspensión del sistema constitucional.

"E. Fuentes Quintana (1975), p. 109.

12 Según éste, la función básica de los aranceles no sería tanto la protección de la producción, sino el aumento de los ingresos fiscales. Puede verse una breve descripción del argumento en P. H. Lindert y C. P. Kindleberger (1982), pp. 147-148. Una aplicación concreta de la tesis se puede encontrar en B. Balassa (1972), pp. 232 y ss. Leticia Alvarez, a quien debo estas referencias, ha aplicado, con un resultado sugestivo aun cuando no concluyente, la misma tesis a la política arancelaria española de la segunda mitad del siglo xix. Cfr. L. Alvarez (1985).

13 J. L. García Delgado y S. Roldón (1974), 2 vols.

14 A pesar de ser un dato bien conocido, sigue siendo uno de los mejores indicios de las características que tuvo la fase expansiva el que el 50 por 100 de las sociedades creadas durante el mismo desaparecieran en la crisis posterior. Cfr. T. Jiménez Araya (1974), p. 177. 
En este marco global, agravado sensiblemente por el ajuste económico que exigían las nuevas condiciones surgidas con la recesión de la postguerra, debe situarse la política presupuestaria de la Dictadura. El cese de los efectos expansivos de la demanda exterior y la subsiguiente pérdida de competitividad de buena parte de los productos más favorecidos por el auge bélico; la crisis bancaria; la reducción espectacular del número de sociedades inscritas; el aumento no menos sensible de la conflictividad; el deterioro de las expectativas empresariales, en una palabra, provocaron la consolidación de lo que se ha denominado la «ideología nacionalista» ${ }^{15}$, que llevaba implícito un elevado grado de intervención estatal en la economía. De ahí que el régimen de Primo de Rivera pueda ser considerado "como medida de urgencia, como solución extrema, [...] para garantizar la continuidad de unos intereses y relaciones sociales gravemente amenazados» ${ }^{16}$. La Dictadura surge, pues, como alternativa ante una confluencia de factores entre los que destacan dos: por un lado, la profunda crisis política de un sistema incapaz de evolucionar integrando a los sectores sociales surgidos como consecuencia de la modernización de España y, por otro, el deterioro de la situación económica y la falta de voluntad de los principales grupos económicos a adaptarse a la nueva coyuntura interior y exterior, a renunciar a mecanismos ajenos al mercado para mantener las tasas de beneficios extraordinarias de los años del conflicto o de reaccionar ante el deterioro de los precios relativos mediante la disminución de los costes medios de producción.

El crecimiento brutal del déficit en los años inmediatamente posteriores al final del conflicto, como muestra el cuadro 1, puede ser considerado la consecuencia de lo anterior. De una media anual de 314 millones de pesetas constantes de 1913 durante el período 1913-1918, el déficit pasó a ser de 576 millones, también de media, en la etapa comprendida entre el ejercicio presupuestario del primer trimestre de 1919 y el de 1922-1923. Un aumento superior al 80 por 100 que implica una tasa de crecimiento acumulativo del 15,1 por 100 .

Tan importante como el aumento del desequilibrio entre gastos e ingresos es la variación en la estructura de los primeros. Como puede comprobarse en el cuadro 2, el déficit creciente fue acompañado de un aumento sustancial de los servicios económicos dentro de la distribución funcional del gasto. Su porcentaje sobre el total pasó del 11 al 18 por 100 , lo cual supone un aumento de más del 50 por 100 (63,6 por 100).

is J. L. García Delgado y S. Roldán (1974), vol. 1, pp. 325.355.

16 J. L. García Delgado (1975), p. 207. 


\section{CUADRO 1}

Saldo presupuestario

(Millones de pesetas)

\begin{tabular}{|c|c|c|c|}
\hline & & Ptas. corr. & Ptas. ctes. \\
\hline $\begin{array}{lllll}1913 & \ldots & \ldots & \ldots & \ldots \\
1914 & \ldots & \ldots & \ldots & \ldots \\
1915 & \ldots & \ldots & \ldots & \ldots \\
1916 & \ldots & \ldots & \ldots & \ldots \\
1917 & \ldots & \ldots & \ldots & \ldots \\
1918 & \ldots & \ldots & \ldots & \ldots \\
1919 & (1 & & \ldots & \text { trimestre } \\
1919-1920 & \ldots & \ldots & \ldots \\
1920-1921 & \ldots & \ldots & \ldots \\
1921-1922 & \ldots & \ldots & \ldots \\
1922-1923 & \ldots & \ldots & \ldots \\
1923-1924 & \ldots & \ldots & \ldots \\
1924 & (2 . & \ldots & \text { trimestre } \\
1924-1925 & \ldots & \ldots & \ldots \\
1925-1926 & \ldots & \ldots & \ldots \\
1926 & (2 . & \text { semestre } \\
1927 & \ldots & \ldots & \ldots & \ldots \\
1928 & \ldots & \ldots & \ldots & \ldots \\
1929 & \ldots & \ldots & \ldots & \ldots\end{array}$ & $\begin{array}{l}\cdots \\
\cdots \\
\cdots \\
\cdots \\
\cdots \\
\cdots \\
\cdots \\
\cdots \\
\cdots \\
\cdots \\
\cdots \\
\cdots \\
\cdots \\
\cdots \\
\cdots \\
\cdots \\
\cdots \\
\cdots\end{array}$ & $\begin{array}{l}-70,7 \\
-166,2 \\
-810,7 \\
-226,9 \\
-965,6 \\
-445,0 \\
-248,5 \\
-1.417,4 \\
-938,1 \\
-1.337,1 \\
-839,8 \\
-667,0 \\
-243,9 \\
-548,6 \\
-492,0 \\
-124,4 \\
-369,4 \\
-164,9 \\
-201,8\end{array}$ & $\begin{array}{l}-70,7 \\
-168,9 \\
-685,3 \\
-160,9 \\
-583,1 \\
-217,2 \\
-121,7 \\
-694,1 \\
-419,9 \\
-724,7 \\
-486,8 \\
-390,3 \\
-134,1 \\
-301,6 \\
-265,9 \\
-71,2 \\
-220,0 \\
-101,4 \\
-120,3\end{array}$ \\
\hline
\end{tabular}

Saldo presupuestario por periodos. Totales y media anual (*)

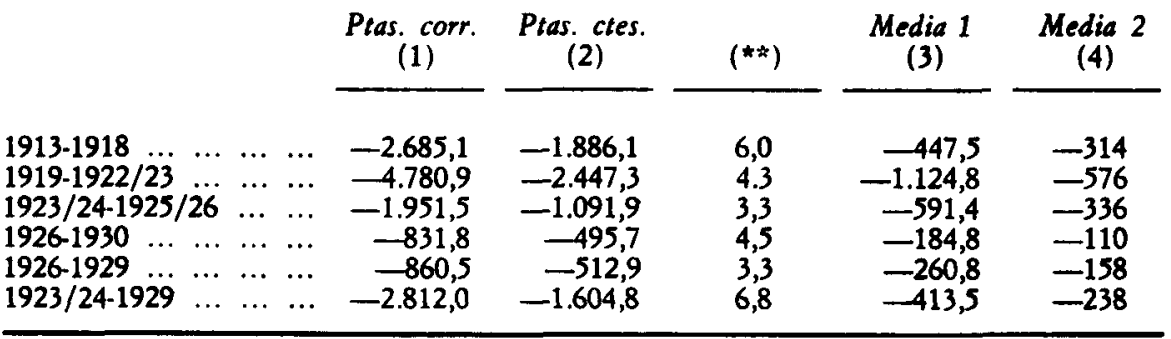

(*) Ingresos ordinarios menos gastos (reconocidos y liquidados).

(**) Factor de conversión: número de meses de los ejercicios presupuestados de cada período considerado dividido por 12 .

Fuentes: Elaborado a partir de P. Martín Aceña (1985), Instituto de Estudios Fiscales (1982) y H. París Eguilez (1943). 


\section{CUADRO 2}

\section{Estructura del gasto del Estado}

(Medias porcentuales)

\begin{tabular}{|c|c|c|c|c|c|}
\hline & Defensa & $\begin{array}{l}\text { Servicios } \\
\text { generales }\end{array}$ & $\begin{array}{l}\text { Servicios } \\
\text { económicos }\end{array}$ & Educación & $\begin{array}{l}\text { Servicios } \\
\text { sociales (*) }\end{array}$ \\
\hline $\begin{array}{lllll}1898-1906 & \ldots & \ldots & \ldots & \ldots \\
1906-1914 & \ldots & \ldots & \ldots & \ldots \\
1914-1918 & \ldots & \ldots & \ldots & \ldots \\
1919-1922 / 23 & \ldots & \ldots & \ldots \\
1923 / 24-1925 / 26 & \ldots & \ldots \\
1926-1930 & \ldots & \ldots & \ldots & \ldots\end{array}$ & $\begin{array}{l}19 \\
23 \\
25 \\
25 \\
29 \\
23\end{array}$ & $\begin{array}{l}12 \\
12 \\
10 \\
11 \\
12 \\
12\end{array}$ & $\begin{array}{r}9 \\
12 \\
11 \\
18 \\
17 \\
14\end{array}$ & $\begin{array}{l}3 \\
4 \\
4 \\
4 \\
4 \\
5\end{array}$ & $\begin{array}{r}11 \\
12 \\
9 \\
8 \\
9 \\
11\end{array}$ \\
\hline
\end{tabular}

(*) Comprende: Educación, Sanidad y Pensiones, S. S. y Beneficencia.

Fuente: Elaborado a partir de Instituto de Estudios Fiscales (1976).

Frente a esta situación, el régimen tenía, en el terreno presupuestario, dos opciones globales básicas: reformar el sistema fiscal, aumentar la eficacia de la administración tributaria, avanzando en la solución del desequilibrio entre gastos e ingresos, o bien proseguir la línea iniciada en los años anteriores consolidando la contribución del Estado en el mantenimiento de los beneficios empresariales.

Una disyuntiva ante la que el principal ministro de Hacienda del régimen se decantaría claramente por la primera opción, como lo demuestra la carta que escribió al dictador a fines de 1925 - y que puede ser considerada como el programa fiscal de la Dictadura-, en la que señalaba de forma tajante que su reforma perseguiría

como fin económico la nivelación del Presupuesto, sin perjuicio de ensanchar los límites del actual para que ninguna zona de las necesidades nacionales quede fuera de sus previsiones y consignaciones; y como objetivo social, modificar la actual distribución de la carga tributaria, con mayor gravamen de las rentas altas ${ }^{17}$.

Como es conocido, poco fue lo que se consiguió en relación con el denominado «objetivo social». Los logros recaudatorios fueron más limitados de lo que pretendió Calvo Sotelo comparando las cifras de finales de los años

17 J. Calvo Sotelo (1930), p. 81. Para llevar a cabo el que denominaba kobjetivo social», en la misma carta planteaba la sustitución de los impuestos de producto por un impuesto progresivo sobre la renta y otro sobre el patrimonio complementario del anterior. 
veinte con las de 1910, obviando, por tanto, la inflación que generó el conflicto de 1914-1918 ${ }^{18}$. Y, a pesar de su intención de reformar la distribución de la carga tributaria, no puede decirse que en 1930 la estructura del sistema fiscal español fuera muy diferente de la existente en 1923. Y ello en especial, y como Calvo Sotelo subrayó, por la negativa tajante de los propietarios a aceptar sus moderadas reformas de enero de $1926^{19}$.

El irrisorio porcentaje que representaban al final de la etapa dictatorial las principales contribuciones directas — prácticamente similar a la media de los dos presupuestos iniciales del régimen- permite valorar la escasa profundidad de una reforma de la que no pudo ni siquiera enorgullecerse su impulsor, tan dado al autoelogio. Lo elevado de los ingresos por Aduanas, por otro lado, permite, además de confirmar la validez de la hipótesis de «gobierno naciente» a la que me he referido, afirmar que debió establecerse una sólida alianza entre los sectores más protegidos y la propia Administración central. Y ello porque un aumento de la protección arancelaria - una vez descartada la posibilidad de poner en práctica la más mínima reforma de la estructura tributaria en sentido progresivo- implicaba no sólo un obstáculo mayor a la entrada de productos extranjeros $y$, por tanto, un mayor mercado -y mayores beneficios- para los empresarios ya instalados, sino también unos ingresos fiscales por este concepto superiores a corto plazo a los derivados de una política arancelaria menos protectora ${ }^{20}$.

En síntesis, medidos en pesetas constantes, los aumentos en la recaudación, aunque no despreciables, no parece que puedan ser calificados de gran progreso, tal y como pretendiera Calvo Sotelo.

\section{III}

Si los logros conseguidos en la recaudación y en la reforma del sistema impositivo no fueron sensibles, tampoco puede decirse que en terreno de la nivelación presupuestaria se hicieran progresos destacados. Lo que sí se llevó a cabo, tanto durante la etapa del Directodio militar como la del Directorio civil, fue aumentar de forma sensible la contribución del Estado al fomento de la actividad de la gran industria, manteniendo un elevado volumen

1 La estratagema de Calvo Sotelo ya fue puesta de relieve por Angel Melguizo. A. Mel. guizo (1979), pp. 430-435.

$19 \mathrm{~J}$. Calvo Sotelo (1930), p. 92.

20 Como es evidente, el aumento de beneficios e ingresos públicos dependerá de la elasticidad de la demanda. Una tarifa muy elevada desincentivaría la importación, de forma que los ingresos disminuirían al no estar gravada la producción en el interior. Y una tarifa demasiado baja fomentaría el aumento de los ingresos fiscales, pero a costa de los beneficios empresariales. 
de déficit. Aun cuando la información disponible no sea todo lo precisa que sería deseable, existen indicadores suficientes para mostrar con contundencia este hecho. El cuadro 2 muestra cómo a partir de 1923 se consolidó el aumento del porcentaje que representaban los servicios económicos dentro del gasto. Lo cual fue acompañado de un aumento notable de la formación bruta de capital fijo, que pasó de un 3 por 100 del gasto total al 5 por 100 en la etapa 1923/24-1925/26, y cerca del 9 por 100 durante el período que transcurre entre el Presupuesto del segundo semestre de 1926 y el de 1929 (cuadro 3). En términos porcentuales, por tanto, la formación bruta de capital fijo - realizada fundamentalmente entre 1926 y 1929 a través del Presupuesto Extraordinario- prácticamente se triplicó entre la etapa predictatorial y el Directorio civil, que fue cuando alcanzó el máximo. De esta forma, si entre $1919-1922 / 23$ y $1923 / 24-1925 / 26$ el aumento fue del 50 por $100(53,47$ por 100), entre este período y el de 1926-1929 llegó prácticamente al 75 por $100(74,80$ por 100$)$.

La estimación de Carreras ${ }^{21}$ de la composición de la formación bruta de capital fijo de la economía española apunta en la misma dirección: la tasa media de crecimiento de la contribución de la inversión pública al GNB entre el quinquenio 1913-1918 y 1927-1929 más que duplicó la de la inversión privada, rozando el 18 por 100 anual (17,92 por 100 ). De esta forma, mientras en el quinquenio señalado la inversión privada era trece veces superior a la inversión pública, en los tres años finales del régimen dictatorial la relación había descendido hasta ser de $5: 1$.

El que los porcentajes en término del gasto total, y todavía más en relación con la Renta Nacional, sean muy moderados no debe de llevar a infravalorar su impacto sobre el ritmo de actividad de algunos sectores. Es imprescindible tener en cuenta que estamos considerando una etapa alejada de la era keynesiana, en la cual el fomento del crecimiento económico no cons-

\section{CUADRO 3}

Porcentaje FBKF en presupuestos sobre gasto total

\begin{tabular}{|c|c|c|c|}
\hline Presupuestos & $\%$ & Presupuestos & $\%$ \\
\hline $\begin{array}{lllllll}1913-1918 & \ldots & \ldots & \ldots & \ldots & \ldots & \ldots \\
1919-1922 / 23 & \ldots & \ldots & \ldots & \ldots & \ldots \\
1923 / 24-1925 / 26 & \ldots & \ldots & \ldots & \ldots\end{array}$ & $\begin{array}{l}3,02 \\
3,31 \\
5,08\end{array}$ & 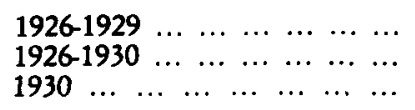 & $\begin{array}{l}8,88 \\
8,14 \\
5,17\end{array}$ \\
\hline
\end{tabular}

FUENTE: La misma del cuadro anterior.

"1 A. Carreras (1985). 
tituía uno de los objetivos centrales de la política económica. Un objetivo que no debe confundirse con el del régimen dictatorial, a pesar de las declaraciones que contienen las exposiciones de motivos de las disposiciones «industrializadoras" promulgadas durante estos años. Estamos, por el contrario, en unos años en los cuales la estabilidad de precios y del cambio exterior y el equilibrio presupuestario configuraban los ejes principales de la actividad económica gubernamental. $Y$, en el caso concreto de España, estamos considerando una economía fundamentalmente agraria, atrasada, no competitiva y con graves dificultades para aumentar la tasa de crecimiento ante las tensiones intersectoriales derivadas de la escasa adaptación de la oferta a los mecanismos típicos de expansión capitalista y el exceso permanente de oferta de mano de obra en el mercado de trabajo, que tendió a mantener los salarios de una parte mayoritaria de la población trabajadora en límites cercanos a los de subsistencia.

En este sentido, en un contexto como aquél, muy diferente del actual, los planteamientos de $\mathrm{M}$. Kalecki de considerar el déficit público como uno de los determinantes de los beneficios empresariales me parecen sugestivos para intentar comprender la función cumplida por la política de gasto dictatorial ${ }^{2}$. En una situación en la cual los trabajadores no ahorran, el saldo de la balanza comercial es poco relevante, el cambio técnico prácticamente nulo y no existen tensiones por el lado de la oferta monetaria ${ }^{23}$, además de la propia inversión privada, el deficit público fomenta la demanda interna, lo cual se traduce - $-y$ en especial si existe capacidad productiva subutilizada y la actividad del sector público se orienta hacia actividades oligopolizadas capitalintensivas-, además de en un aumento del empleo industrial ${ }^{24}$, en el crecimiento de los beneficios del conjunto de los capitalistas del sector de bienes de inversión. $Y$ parece posible afirmar que estas condiciones que se acaban de mencionar se daban en la economía española durante los años veinte.

Acabo de referirme a la importancia de un déficit creciente como factor

${ }^{22}$ Un esbozo de los mismos en J. Palafox (1980).

${ }^{3}$ P. Martín Aceña (1984), pp. 157 y ss. Aunque, como he señalado, los aspectos monetarios quedan fuera de estas páginas, no deja de ser sorprendente que, en el sugestivo análisis IS-LM desarrollado por Pablo Martín Aceña, el kefecto riqueza» del déficit financiado con deuda sea escasamente considerado, a pesar de que se le reconozca un efecto expansivo sustancial (pp. 125 y ss.).

${ }^{24}$ En España, el crecimiento del número de trabajadores industriales estuvo sometido a dos tendencias contrapuestas. Por un lado, la ausencia de innovación técnica tendió a hacerlos aumentar al hacerlo la inversión agregada. Pero, por otro lado, las condiciones de los principales sectores beneficiados por la política gubernamental tuvo que frenar su expansión. Los datos de Alcaide (1976) y Carreras (1984) permiten estimar que frente a un estancamiento de la productividad industrial, o incluso un ligero descenso si se adopta el valor añadido de Alcaide como aproximación, la población activa del sector secundario aumentó en un 31,9 por 100 entre 1923 y 1929 . Las implicaciones de este estancamiento técnico, desde la perspectiva de qué características tuvo la expansión industrial española entre 1923 y 1930, son obvias. 
de expansión de los beneficios sin haber hecho referencia a su evolución. $\mathrm{Y}$, en ese sentido, si se consideran los resultados que aparecen en la segunda parte del cuadro 1 (Saldo presupuestario por períodos. Totales y media anual), es evidente que éstos no confirman lo que se acaba de exponer. Mientras, como ya he mencionado, el déficit medio anual de la etapa 1919-1922/23 fue de 576 millones de pesetas constantes de 1913; entre 1923/24-1925/26 descendió a 335 millones anuales, disminuyendo todavía más durante la etapa en la que Calvo Sotelo estuvo al frente del Ministerio de Hacienda.

Según estos resultados, estaríamos ante una situación opuesta a la que he planteado en los párrafos anteriores, dado que la disminución del déficit lleva a una contracción de la cuantía de los beneficios empresariales determinada por la acción del sector público central. A pesar, por tanto, del Presupuesto Extraordinario, Primo de Rivera habría llevado a cabo una política muy poco relevante desde la perspectiva de estos sectores.

Considerar la política presupuestaria a partir de los resultados del cuadro 1, que es lo que hacen Francisco Comin y Pablo Martín Aceña, supone, en mi opinión, infravalorar su función y no considerar los diversos mecanismos cuasi-presupuestarios que se emplearon para favorecer a los sectores de la gran industria. Supone aislar algunos elementos de la política de gasto, en aras de un mayor rigor contable, haciendo imposible una valoración completa de ésta.

$\mathrm{Y}$ ello por dos razones fundamentales que, a su vez, eliminan la contradicción aparente a la que me he referido. En primer lugar, porque si bien desde la perspectiva de los beneficios empresariales lo significativo es el déficit, para los empresarios de los sectores de bienes de inversión -principales beneficiarios de la política presupuestaria- lo relevante es la variación del gasto con efectos inducidos sobre su actividad. $\mathrm{Y}$, en segundo lugar, porque, a través de mecanismos heterodoxos, la Dictadura impulsó, o aceptó, un elevado volumen de gasto no realizado a través de los Presupuestos del Estado, pero que finalmente repercutieron sobre ellos.

En relación con la primera cuestión, lo relevante, en mi opinión, no es, en modo alguno, el porcentaje que representan determinadas magnitudes presupuestarias sobre la Renta Nacional, que en aquellos años tenían que ser necesariamente muy modestos. Lo que permite comprender y valorar la actuación de la Dictadura en este terreno es en qué medida apoyó financieramente a determinados sectores, con independencia de que contablemente éstos constaran en el Presupuesto, y cuál fue el coste de oportunidad sobre el conjunto de la economía.

$\mathrm{Y}$, como puede comprobarse en el cuadro $5^{25}$, el gasto estatal en ferro-

${ }^{25}$ Cuyo detalle puede verse en el cuadro 4. 


\section{CUADRO 4}

Estimación del gasto durante el Directorio civil (En millones de pesetas y porcentajes)

\begin{tabular}{|c|c|c|c|c|c|c|c|c|}
\hline & 1926 & & 1927 & & 1928 & & 1929 & \\
\hline \multicolumn{9}{|l|}{ P. Ordinario } \\
\hline $\begin{array}{lll}1 & \text { Tot. Carreteras . . .. } \\
2 & \text { Tot. FF. CC. ‥ } & \ldots \\
3 & \text { Ser. Económicos } & \ldots\end{array}$ & $\begin{array}{r}95,3 \\
17,3 \\
240,3\end{array}$ & & $\begin{array}{r}183,8 \\
25,3 \\
240,1\end{array}$ & & $\begin{array}{r}164,8 \\
26,0 \\
491,8\end{array}$ & & $\begin{array}{r}163,3 \\
33,6 \\
528,6\end{array}$ & \\
\hline \multicolumn{9}{|l|}{ P. Extraordinario } \\
\hline $\begin{array}{llllll}4 & \text { Tot. Carreteras } & \ldots \\
5 & \text { P. Ex. Fomento } & \ldots \\
6 & 3+5 & \ldots & \ldots & \ldots & \ldots\end{array}$ & $\begin{array}{r}2,5 \\
16,3 \\
256,6\end{array}$ & & $\begin{array}{r}84,0 \\
166,8 \\
563,1\end{array}$ & & $\begin{array}{l}163,9 \\
265,2 \\
655,7\end{array}$ & & $\begin{array}{l}198,5 \\
305,8 \\
727,1\end{array}$ & \\
\hline \multicolumn{9}{|l|}{ Caja Ferroviaria } \\
\hline \multirow[t]{2}{*}{7 Deuda + R. Ced. ... } & 326,8 & & 169,9 & & 195,7 & & 467,4 & \\
\hline & & $\%(*)$ & & $\%$ & & $\%$ & & $\%$ \\
\hline \multicolumn{9}{|l|}{ Totales } \\
\hline $\begin{array}{l}3+5+7 \text { Tot. G. Ec. } \\
1+4 \text { Carreteras . . . } \\
2+7 \text { FF. CC. } \ldots . . . \\
1+2+4+7 \text { Tot. Tr. }\end{array}$ & $\begin{array}{r}583,2 \\
97,8 \\
344,1 \\
441,9\end{array}$ & $\begin{array}{r}100,00 \\
16,76 \\
59,00 \\
75,77\end{array}$ & $\begin{array}{l}733,0 \\
267,8 \\
195,2 \\
463,0\end{array}$ & $\begin{array}{r}100,00 \\
36,53 \\
26,63 \\
63,16\end{array}$ & $\begin{array}{l}851,4 \\
328,7 \\
221,7 \\
550,4\end{array}$ & $\begin{array}{r}100,00 \\
38,61 \\
26,03 \\
64,64\end{array}$ & $\begin{array}{r}1.194,0 \\
361,8 \\
500,9 \\
862,7\end{array}$ & $\begin{array}{r}100,00 \\
30,30 \\
41,95 \\
72,25\end{array}$ \\
\hline
\end{tabular}

(*) Porcentaje sobre el gasto económico total.

Fuentes: Instituto de Estudios Fiscales (1976); Dirección General de Tesorería y Contabilidad, Liquidación del Presupuesto Extraordinario, y J. Velarde (1968).

carriles y carreteras, cuyos efectos inducidos sobre las industrias de bienes de inversión son evidentes, aumentó durante el régimen autoritario. $\mathrm{Y}$ si lo hizo de forma sensible dentro del presupuesto ordinario, lo hizo de forma espectacular a través de diferentes caminos que no pueden ser considerados ajenos a la política presupuestaria de gasto, aunque su utilización le permitiera al régimen, y en especial a Calvo Sotelo, vanagloriarse de haber resuelto el problema del déficit. A partir del presupuesto de 1921-22, como puede verse en la columna 4, el gasto en carreteras y ferrocarriles pasó a ser más de la mitad del gasto total en Servicios Económicos del presupuesto ordinario, aumentando sustancialmente en relación con años anteriores. Un crecimiento que es paralelo al aumento del peso de los Servicios Económicos dentro del gasto del presupuesto ordinario, lo cual supuso una fuerte expansión en términos absolutos, que queda reflejada en la columna 2. 


\section{CUADRO 5}

Gasto en carreteras y ferrocarriles de la Administración central (Millones de pesetas)

\begin{tabular}{|c|c|c|c|c|c|c|c|c|c|c|c|}
\hline & & & & (1) & (2) & (3) & (4) & (5) & (6) & (7) & (8) \\
\hline $\begin{array}{lll}1913 & \ldots & \ldots \\
1914 & \ldots & \ldots \\
1915 & \ldots & \ldots \\
1916 & \ldots & \ldots \\
1917 & \ldots & \ldots \\
1918 & \ldots & \ldots \\
1919 & \ldots & \ldots \\
1919-1920 & \ldots \\
1920-1921 & \ldots \\
1921-1922 & \ldots \\
1922-1923 & \ldots \\
1923-1924 & \ldots \\
1924 & \ldots & \ldots \\
1924-1925 & \ldots \\
1925-1926 & \ldots \\
1926 & \ldots & \ldots \\
1927 & \ldots & \ldots \\
1928 & \ldots & \ldots\end{array}$ & $\begin{array}{c}\ldots \\
\ldots \\
\ldots \\
\ldots \\
\ldots \\
\ldots \\
\ldots \\
\ldots \\
\ldots \\
\ldots \\
\ldots \\
\ldots \\
\ldots \\
\ldots \\
\ldots \\
\ldots \\
\ldots\end{array}$ & $\begin{array}{l}\ldots \\
\ldots \\
\ldots \\
\ldots \\
\ldots \\
\ldots \\
\ldots \\
\ldots \\
\ldots \\
\ldots \\
\ldots \\
\ldots \\
\ldots \\
\ldots \\
\ldots \\
\ldots \\
\ldots \\
\ldots\end{array}$ & $\begin{array}{l}\ldots \\
\ldots \\
\ldots \\
\ldots \\
\ldots \\
\ldots \\
\ldots \\
\ldots \\
\ldots \\
\ldots \\
\ldots \\
\ldots \\
\ldots \\
\ldots \\
\ldots \\
\ldots \\
\ldots \\
\ldots\end{array}$ & $\begin{array}{r}175,6 \\
188,5 \\
198,8 \\
201,3 \\
210,3 \\
222,0 \\
78,0 \\
432,8 \\
776,2 \\
674,1 \\
729,8 \\
616,7 \\
166,4 \\
553,1 \\
622,1 \\
240,4 \\
479,3 \\
491,9\end{array}$ & $\begin{array}{r}69,5 \\
76,3 \\
79,0 \\
83,0 \\
90,3 \\
87,7 \\
22,0 \\
115,2 \\
219,3 \\
361,3 \\
395,8 \\
326,9 \\
95,1 \\
276,0 \\
332,7 \\
113,0 \\
209,1 \\
190,3\end{array}$ & $\begin{array}{r}69,5 \\
76,3 \\
79,0 \\
83,0 \\
90,3 \\
87,7 \\
22,0 \\
115,2 \\
219,3 \\
361,3 \\
395,8 \\
326,9 \\
95,1 \\
276,0 \\
332,7 \\
442,3 \\
463,0 \\
549,9\end{array}$ & $\begin{array}{l}39,6 \\
40,5 \\
39,7 \\
41,2 \\
42,9 \\
39,5 \\
28,2 \\
26,6 \\
28,3 \\
53,6 \\
54,2 \\
53,0 \\
57,2 \\
49,9 \\
53,5 \\
47,0 \\
43,6 \\
38,7\end{array}$ & $\begin{array}{r}39,6 \\
40,5 \\
39,7 \\
41,2 \\
42,9 \\
39,5 \\
28,2 \\
26,6 \\
28,3 \\
53,6 \\
54,2 \\
53,0 \\
57,2 \\
49,9 \\
53,5 \\
184,0 \\
96,6 \\
111,8\end{array}$ & $\begin{array}{r}88,1 \\
94,5 \\
99,7 \\
101,0 \\
105,5 \\
111,3 \\
39,1 \\
217,1 \\
389,3 \\
338,1 \\
366,0 \\
309,3 \\
83,5 \\
277,4 \\
312,0 \\
120,6 \\
240,4 \\
246,7\end{array}$ & $\begin{array}{r}85,8 \\
94,2 \\
97,6 \\
102,5 \\
111,5 \\
108,3 \\
27,2 \\
142,3 \\
270,9 \\
446,3 \\
488,9 \\
403,8 \\
117,5 \\
340,9 \\
410,9 \\
139,6 \\
258,3 \\
235,1\end{array}$ & $\begin{array}{r}85,8 \\
94,2 \\
97,6 \\
102,5 \\
111,5 \\
108,3 \\
27,2 \\
142,3 \\
270,8 \\
446,2 \\
488,8 \\
403,7 \\
117,4 \\
340,8 \\
410,8 \\
546,2 \\
471,7 \\
679,1\end{array}$ \\
\hline
\end{tabular}

(1) Servicios económicos.

(2) Gasto en carreteras y ferrocarril según el presupuesto ordinario.

(3) Gasto estimado en carreteras y ferrocarril a partir del cuadro 4.

(4) $2 / 1$.

(5) $3 / 1$.

(6) Indice base 100 para la media 1913-1918 de 1.

(7) Indice base 100 para la media 1913-1918 de 2.

(8) Indice base 100 para la media 1913-1918 de 3.

FUENTES: Las mismas que el cuadro 4.

La disminución del porcentaje en los años finales que se observa en la columna 4 no puede llevar a confusión. Coincide con los años de funcionamiento de lo que podrían denominarse mecanismos heterodoxos de financiación, a través de los cuales, como muestran las columnas 5 y 8 , se canalizaron la mayor parte de las ayudas.

Pero es que, además, y en relación con la segunda de las cuestiones apuntadas, el régimen utilizó ampliamente un instrumento cuasi-presupuestario para incrementar las ganancias de los sectores de bienes de inversión. Así, además de otros aspectos de la política económica no menos relevantes o de la represión sindical, en estos años se utilizó ampliamente la concesión del 
aval del Estado para emisiones de títulos de renta fija destinadas a la realización de inversiones «reproductivas». Inversiones que resultaron ser tan poco «reproductivas» que el Estado -el Estado republicano, cuya participación en los proyectos fue obviamente nula - tuvo que hacerse cargo del pago de intereses y de su amortización. Inversiones privadas financiadas con el aval del Estado, que fue quien al final tuvo que hacer frente a las obligaciones derivadas de su condición de avalista, que deben de ser tenidas en cuenta para estimar el déficit real durante la dictadura del general Primo de Rivera, puesto que fue entonces cuando se realizaron las emisiones y fue entonces, también, cuando tuvieron lugar sus efectos sobre el mercado financiero.

De esta forma, entre 1923 y 1930 se emitieron $1.461,3$ millones de pesetas en títulos avalados por el Estado, que fueron incluidos en los Presupuestos de 1934 y 1935 . El detalle de éstos muestra cómo la inmensa mayoría correspondian a la Deuda Especial Ferroviaria (1.273,5 millones). El que no fuera emitida como Deuda del Estado para permitir compaginar el cumplimiento del objetivo central de la política presupuestaria - la ayuda a los sectores de la gran industria - con la no ampliación de la cuantía declarada del déficit no impide que haya que considerarla como tal. Y como Deuda del Estado debe de ser computada en el cálculo del déficit con el que se saldaron los presupuestos, por más que no constara en éstos. Lo mismo debe de hacerse con las obligaciones de la Compañía Trasmediterránea y el resto de las emisiones de la etapa dictatorial que fueron incluidas dentro de la Deuda del Estado en circulación en los años de la II República.

El resumen de esta operación es el que aparece en el cuadro 7. La comparación entre los saldos medios del cuadro 6 , en el que se han corregido los saldos del cuadro 1 , con los resultados del cuadro 7 permite subrayar la infravaloración del déficit en que se incurre atendiendo únicamente a los discutibles presupuestos, ordinarios y extraordinarios, confeccionados. Aunque la cuantía media del mismo disminuya en ambos casos en relación con la etapa 1919-1922/23, puede comprobarse cómo, en pesetas constantes, los resultados son muy diferentes. En el cuadro 1, el saldo negativo medio anual de la etapa 1923/24-1925/26 es prácticamente similar al de 1913-1918. En el cuadro 6, por el contrario, éste supera a aquél en un 37 por 100. La disparidad es todavía mayor para la etapa 1926-1929 (ó 1926-1930), ya que fue entonces cuando el mecanismo mencionado funcionó con mayor intensidad. El saldo negativo más que se duplica al tomar en consideración la Deuda avalada por el Estado, superando ligeramente el déficit medio del período 1913. 1918 si se consideran exclusivamente los ejercicios presupuestarios gestionados por la Dictadura ${ }^{26}$.

26 Esto es, desde el del segundo semestre de 1926 al Presupuesto anual de 1929. Como es bien conocido, 1930 estuvo marcado, en el terreno de política presupuestaria, por una 


\section{CUADRO 6}

Saldo presupuestario corregido

(Millones de pesetas)

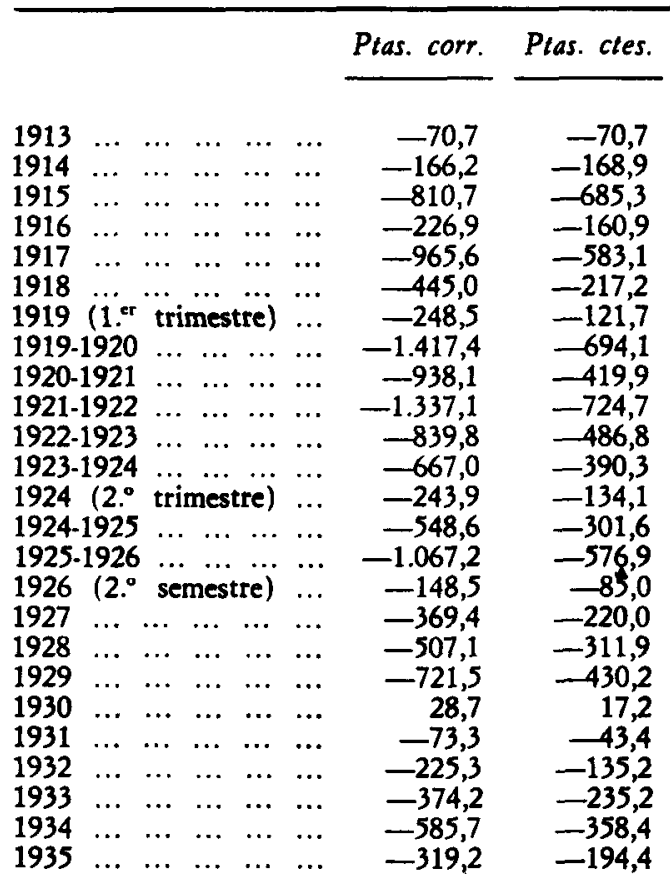

Corrección Saldo presupuestario por periodos. Totales y media anual

\begin{tabular}{|c|c|c|c|c|c|}
\hline & $\begin{array}{c}\text { Ptas corr. } \\
\text { (1) }\end{array}$ & $\begin{array}{c}\text { Ptas. ctes. } \\
\text { (2) }\end{array}$ & F. $c$. & $\underset{(3)}{\text { Media } 1}$ & $\begin{array}{c}\text { Media } 2 \\
\text { (4) }\end{array}$ \\
\hline $\begin{array}{lllll}1913-1918 & \ldots & \ldots & \ldots & \ldots \\
1919-1922 / 23 & \ldots & \ldots & \ldots \\
1923 / 24-1925 / 26 & \ldots & \ldots \\
1926-1930 & \ldots & \ldots & \ldots & \ldots \\
1926-1929 & \ldots & \ldots & \ldots & \ldots \\
1923 / 24-1929 & \ldots & \ldots & \ldots \\
1931-1935 & \ldots & \ldots & \ldots & \ldots\end{array}$ & $\begin{array}{l}-2.685,1 \\
-4.780,9 \\
-2.526,7 \\
-1.717,8 \\
-1.746,5 \\
-4.273,2 \\
-1.577,7\end{array}$ & $\begin{array}{r}-1.886,1 \\
-2.447,3 \\
-1.402,8 \\
-1.029,9 \\
-1.047,1 \\
-2.449,9 \\
-966,7\end{array}$ & $\begin{array}{l}6,0 \\
4,3 \\
3,3 \\
4,5 \\
3,3 \\
6,8 \\
5,0\end{array}$ & $\begin{array}{r}-447,5 \\
-1.124,9 \\
-777,4 \\
-381,7 \\
-537,4 \\
-633,1 \\
-315,5\end{array}$ & $\begin{array}{l}-314,3 \\
-575,8 \\
-431,6 \\
-228,9 \\
-322,2 \\
-362,9 \\
-193,3\end{array}$ \\
\hline
\end{tabular}

FuenTES: Las mismas del cuadro 1 e Instituto de Estudios Fiscales (1976).

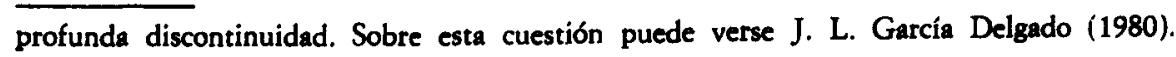




\section{CUADRO 7}

Déficit presupuestario total

(Millones de pesetas)

\begin{tabular}{|c|c|c|c|c|c|c|c|}
\hline & & & & (1) & (2) & (3) & \\
\hline 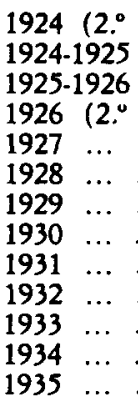 & \begin{tabular}{ccc}
\multicolumn{3}{c}{ trimestre) } \\
$\ldots$ & $\ldots$ & $\ldots$ \\
$\ldots$ & $\ldots$ & $\ldots$ \\
semestre) \\
$\ldots$ & $\ldots$ & $\ldots$ \\
$\ldots$ & $\ldots$ & $\ldots$ \\
$\ldots$ & $\ldots$ & $\ldots$ \\
$\ldots$ & $\ldots$ & $\ldots$ \\
$\ldots$ & $\ldots$ & $\ldots$ \\
$\ldots$ & $\ldots$ & $\ldots$ \\
$\ldots$ & $\ldots$ & $\ldots$ \\
$\ldots$ & $\ldots$ & $\ldots$ \\
$\ldots$ & $\ldots$ & $\ldots$ \\
$\ldots$ & $\ldots$ & $\ldots$ \\
$\ldots$ & $\ldots$ & $\ldots$
\end{tabular} & $\begin{array}{l}\ldots \\
\ldots \\
\cdots \\
\cdots \\
\cdots \\
\cdots \\
\cdots \\
\cdots \\
\cdots \\
\cdots \\
\cdots \\
\cdots \\
\cdots\end{array}$ & $\begin{array}{r}-243,9 \\
-548,6 \\
-492 \\
-124,4 \\
-369,4 \\
-164,9 \\
-201,8 \\
28,7 \\
-73,3 \\
297,4 \\
-374,2 \\
-585,7 \\
-319,2\end{array}$ & $\begin{array}{l}290,4 \\
497\end{array}$ & 20,8 & $\begin{array}{l}88,7 \\
24,1 \\
31 \\
22,7\end{array}$ & $\begin{array}{r}-243,9 \\
-548,6 \\
-1.067,2 \\
-148,5 \\
-369,4 \\
-507,1 \\
-721,5 \\
28,7 \\
-73,3 \\
297,4 \\
-374,2 \\
-585,7 \\
-319,2\end{array}$ \\
\hline
\end{tabular}

(1) Deuda de la Caja Ferroviaria incluida en la Deuda del Estado en circulación en el ejercicio de 1934.

(2) Obligaciones hipotecarias de Ferrocarriles Madrid-Cáceres-Portugal ( 5 millones) y obligaciones hipotecarias Ferrocarriles Plasencia-Astorga incluidas en la Deuda del Estado en circulación en el ejercicio de 1935.

(3) Corresponden a obligaciones de la Compañía Trasmediterránea, excepto 22,7 millones de 1929 que corresponden a obligaciones del Patronato Nacional de Turismo, incluidas en la Deuda del Estado en circulación en el ejercicio de 1924.

Nota: Las emisiones se han asignado al ejercicio presupuestario en que fueron puestas en circulación.

Fuentes: Las mismas del cuadro 1 e Instituto de Estudios Fiscales (1976).

La contrapartida de esta ocultación de la cuantía real del déficit es la necesidad de revisar las cifras de Deuda Pública emitida y en circulación durante el período. Como es evidente, si se procede a restar de los ejercicios republicanos las emisiones realizadas entre 1923 y 1929, pero incorporadas a las obligaciones del Estado en los años treinta, el resultado es sensiblemente diferente al ofrecido por Francisco Comín y Pablo Martín Aceña, para quienes parece claro que la deuda total en circulación (Estado, Tesoro y especiales interiores) aumentó más entre 1930 y 1935 que entre 1923 y $1930^{\pi}$.

Como puede deducirse de los cuadros 8 y 9 , durante la etapa en la que Calvo Sotelo estuvo al frente de la Hacienda el aumento real de la Deuda del Estado en circulación fue el mismo que entre 1923-24 y 1925-26: $2.941 \mathrm{mi}$ llones de pesetas, lo que representa una tasa de crecimiento acumulativo anual

${ }^{27}$ F. Comín Comín y P. Martín Aceña (1984), p. 253. 


\section{CUADRO 8}

Aumento deuda a cargo del Estado en circulación

(Millones de pesetas)

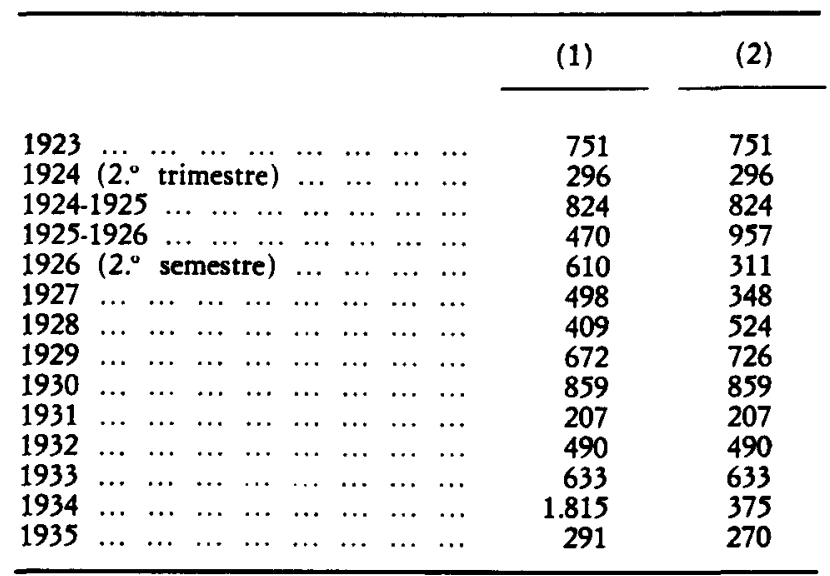

(1) F. Comín y P. Martín Aceña (1984).

(2) Se han restado en 1934 y 1935 las deudas especiales incluidas en esos ejercicios correspondientes a los años de la Dictadura, incluyéndose en los años de emisión real. Puede verse el detalle en el cuadro 7.

Nota: Deuda Pública del Tesoro y especiales.

Fuentes: Col. 1, F. Comín y P. Martín Aceña (1984); col. 2, Instituto de Estudios Fiscales (1976).

inferior a la del Directorio militar (5,1 y 6,7 por 100$)$, pero muy superior a la de los dos períodos republicanos tradicionales, en los que fue de 2,3 por 100 para la etapa republicano-socialista y de 1,7 por 100 para el bienio negro.

Más ilustrativas que las tasas de crecimiento acumulado, que están influidas por el aumento del divisor, son, a mi juicio, las cifras medias anuales recogidas en el cuadro 9. Como puede comprobarse, la cifra media de aumento anual de la deuda real en circulación fue muy superior durante la Dictadura que durante la II República (864 millones frente a 395), y dentro de la etapa del régimen autoritario no se observan diferencias apreciables entre unos períodos y otros. $\mathrm{Y}$ aun cuando la valoración de las implicaciones de estos resultados sobre las diferencias de la política fiscal de ambos regímenes queda fuera del marco fijado para estas páginas, no está fuera de lugar insistir en el profundo contraste que suponen. 


\section{CUADRO 9}

\section{Deuda total emitida durante la Dictadura y la II República}

\begin{tabular}{|c|c|c|c|c|c|}
\hline & \multicolumn{2}{|c|}{ Total } & \multirow[b]{2}{*}{$(*)$} & \multicolumn{2}{|c|}{ Media anual } \\
\hline & (1) & (2) & & (1) & (2) \\
\hline \multirow[t]{3}{*}{ 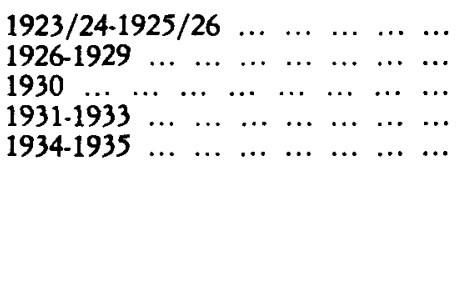 } & $\begin{array}{r}2.341 \\
2.189 \\
859 \\
1.330 \\
2.106\end{array}$ & $\begin{array}{r}2.941 \\
2.941 \\
859 \\
1.330 \\
645\end{array}$ & $\begin{array}{l}3,3 \\
3,5 \\
1,0 \\
3,0 \\
2,0\end{array}$ & $\begin{array}{r}709 \\
625 \\
859 \\
443 \\
1.053\end{array}$ & $\begin{array}{l}891 \\
840 \\
859 \\
443 \\
322\end{array}$ \\
\hline & \multicolumn{2}{|c|}{ Total } & & \multicolumn{2}{|c|}{ Media anual } \\
\hline & (1) & (2) & & (1) & (2) \\
\hline $\begin{array}{lllll}\text { Emitida } & \text { Dictadura }\left(^{*}\right) & \ldots & \ldots & \ldots \\
\text { Emitida } & \text { II República } & \ldots & \ldots & \ldots\end{array}$ & $\begin{array}{l}5.389 \\
3.436\end{array}$ & $\begin{array}{l}6.741 \\
1.975\end{array}$ & & $\begin{array}{l}690 \\
687\end{array}$ & $\begin{array}{l}864 \\
395\end{array}$ \\
\hline
\end{tabular}

(*) Factor de conversión. Véase cuadro 1.

(1) Según cálculo de F. Comín y P. Martín Aceña (1984).

(2) Según cuadro 8.

FuenTES: Las mismas del cuadro 8.

\section{IV}

Teniendo en cuenta lo escrito hasta aquí, no puede sorprender que la política presupuestaria, y más en concreto de gasto público, llevada a cabo por la dictadura del general Primo de Rivera deba de ser valorada negativamente. Si en relación con los ingresos realizó escasos avances en la modernización del sistema fiscal, en el lado del gasto sus limitaciones fueron todavía más evidentes. Por una parte, rompió el principio de unidad presupuestaria poniendo en funcionamiento mecanismos no sujetos a control. Por otra, ni reordenó significativamente la cuantía de la Deuda en circulación —aunque la conversión llevada a cabo por Calvo Sotelo sea uno de los pocos aspectos técnicamente positivos de su gestión- ni disminuyó de forma apreciable el déficit público, aunque lo ocultara. Ninguno de los dos objetivos centrales del principal ministro de Hacienda del régimen, por tanto, fue cumplido.

Lo que sí llevó a cabo fue una destacable operación de enmascaramiento de los gastos realizados de manera que un análisis estricto de las cuentas ge. nerales del Estado permitiera ofrecer unos resultados muy diferentes a los reales. Extrayendo de los presupuestos partidas nada despreciables del gasto 
total, el régimen intentó $-\mathrm{y}$ consiguió en alguna medida - justificar unas realizaciones inexistentes. La ocultación del saldo negativo con que se cerraron los Presupuestos Generales, al presentar exclusivamente los resultados del ordinario, no es, desde esta perspectiva, el rasgo más destacado.

Junto a esta tosca decisión, se adoptaron decisiones mucho más graves que comprometieron la gestión de los fondos públicos - según los criterios de la ortodoxia vigente- en los años siguientes. La creación de las Cajas Especiales, y especialmente de la Caja Ferroviaria, que, según los datos disponibles, fue la que contó con un volumen de fondos más cuantioso, financiadas prácticamente de forma exclusiva con fondos avalados por el Estado, permitió, como he señalado, aumentar el gasto ocultándolo a cualquier tipo de control.

Y esta ausencia de control es quizá lo más criticable por cuanto agudizó los numerosos y graves problemas de la economía española. Las inversiones denominadas reproductivas, que fueron justificadas en base a que su rentabilidad generaría los fondos suficientes para su amortización, industrializando, además, al país, fueron realizadas sin ningún tipo de análisis sobre su viabilidad y sin exigir ninguna contrapartida de mejora técnica a los sectores más beneficiados por ellas. De esta forma, una vez desaparecido el régimen dictatorial, el Estado tuvo que hacerse cargo de las obligaciones contraídas, disminuyendo el margen de maniobra para ejecutar una política presupuestaria diferente. $Y$ los sectores que habían visto aumentada su demanda entraron en una etapa de crisis, con evidentes repercusiones sobre la estabilidad política del régimen republicano.

Por otro lado, los resultados del cuadro 10 y el que, como he señalado, el estancamiento técnico fuera una de las características del sector industrial durante estos años, refuerzan la valoración negativa que se acaba de apuntar y, a mi juicio, confirman la tesis de que la política fiscal desarrollada por Primo de Rivera no hizo sino consolidar el «capitalismo asistido» que caracteriza a la evolución de la economía española, y de forma muy especial a lo que he denominado el sector de la gran industria, durante el primer tercio del siglo $\mathbf{x x}$.

Las diferentes relaciones funcionales establecidas entre la inversión pública, la inversión privada y diversos indicadores de actividad confirman la relevancia de la primera en la evolución de algunos de los sectores de mayor peso dentro de la gran industria. Así, su elasticidad pasa de ser no significativa en la regresión número 1 - en la que se relacionan ambas con el Gasto Nacional Bruto- ${ }^{26}$ a ser apreciable cuando se consideran sectores industriales incluidos dentro de la definición de gran industria.

a El resultado de esta regresión, en la que ni la inversión privada ni la realizada por el sector público son significativas, podría tener múltiples explicaciones, entre las cuales 
CUADRO 10

Elasticidad de las inversiones privada y pública respecto a las variables que se indican (1920-1935)

\begin{tabular}{|c|c|c|c|c|c|c|}
\hline & $\begin{array}{c}\text { Variable } \\
\text { dependiente }\end{array}$ & $\begin{array}{l}\text { Inversión } \\
\text { privada }\end{array}$ & $\begin{array}{l}\text { Inversión } \\
\text { pública }\end{array}$ & $R$ & $D-W$ & $F$ \\
\hline $\begin{array}{lllllll}(1) & \ldots & \ldots & \ldots & \ldots & \ldots\end{array}$ & GNB & $\begin{array}{r}0,0874 \\
(1,1233)\end{array}$ & $\begin{array}{r}0,0908 \\
(2,3588)\end{array}$ & 0,36 & 2,9 & 3,8 \\
\hline $\begin{array}{lllllll}(2) & \ldots & \ldots & \ldots & \ldots & \ldots\end{array}$ & IPI & $\begin{array}{r}0,2684 \\
(3,9326)\end{array}$ & $\begin{array}{r}0,2056 \\
(6,088)\end{array}$ & 0,82 & 1,4 & 29,7 \\
\hline $\begin{array}{lllllll}(3) & \ldots & \ldots & \ldots & \ldots & \ldots\end{array}$ & IPCEM & $\begin{array}{r}1,4049 \\
(10,9846)\end{array}$ & $\begin{array}{r}0,217 \\
(3,4298)\end{array}$ & 0,91 & 2,02 & 72,0 \\
\hline $\begin{array}{lllllll}(4) & \ldots & \ldots & \ldots & \ldots & \ldots\end{array}$ & IPACE & $\begin{array}{r}1,0611 \\
(9,1881)\end{array}$ & $\begin{array}{r}0,3648 \\
(6,3860)\end{array}$ & 0,91 & 1,26 & 71,0 \\
\hline
\end{tabular}

GNB = Gasto Nacional Bruto.

IPI = Indice de Producción Industrial.

IPCEM = Indice de Producción de Cemento.

IPACE = Indice de Producción de Acero.

Fuentes: GNB, IPI, inversión pública y privada, Carreras (1984) y (1985); IPACE, Barreiro Zabala (s. a.: ¿1943?); IPCEM, CECA (1975).

Lo que sí consiguió el régimen dictatorial, por tanto, fue favorecer a los sectores dominantes, cuyo apoyo en la suspensión del sistema político restauracionista no puede considerarse escaso. Y, a través de los presupuestos -que constituyen sólo uno de los ejes de la política económica articulada ${ }^{29}$-, los mayores beneficiarios fueron los sectores a los que me he referido. Fueron ellos los que consiguieron una proporción mayor de los fondos destinados a servicios económicos dentro del presupuesto ordinario; fueron los que obtuvieron las mayores ventajas de la puesta en funcionamiento del presupuesto extraordinario, y fueron ellos también los grandes beneficiarios de la actuación de las Cajas Especiales. Incapaces de expander su producción con costes decrecientes a un ritmo suficiente para acortar la diferencia que los separaba de los precios exteriores, incapaces de racionalizar su actividad con criterios mínimos de eficiencia, tuvieron en la política presupuestaria un mecanismo óptimo a corto y medio plazo para aumentar sus tasas de beneficios. Las consecuencias negativas de todo ello se harían evidentes a partir del mismo momento en que Primo de Rivera fue obligado a abandonar el poder.

no es la menos improbable que la estimación de Carreras esté infravalorando ambas. No es posible descartar, sin embargo, que el concepto de inversión, tal y como lo defínimos en la actualidad, tenga menor relevancia de la que a menudo se le concede en una economía agraria y atrasada desde el punto de vista técnico.

2 Una excelente síntesis de la cual puede encontrarse en J. L. García Delgado (1983). 


\section{BIBLIOGRAFIA}

AlCaide (1976): «Una revisión urgente de la serie de renta nacional española en el siglo XX*, en Instituto de Estudios Fiscales: Datos básicos para la Historia Financiera de España, 1850-1975, Madrid, Instituto de Estudios Fiscales, vol. I, pp. 1126-1150.

Alvarez, L. (1985): La politica comercial española, 1849-1891 (tesis de licenciatura), Valencia, Facultad de Ciencias Económicas y Empresariales.

BaIRoch, P. (1976): «Europe's Gross National Product: 1800-1975», Journal of European Economic History, núm. 2, pp. 273-340.

Barreiro Zabala (s. a.: ¿1943?): Estadística minero-siderúrgica de España por..., Madrid.

Calvo Sotelo, J. (1930): Mis servicios al Estado, Madrid (Instituto de Estudios de la Administración Local, Madrid, 1974).

Carreras, A. (1984): «La producción industrial española, 1842-1981: construcción de un índice anual», Revista de Historia Económica, II, núm. 1, pp. 127.157.

- (1985): *Gasto nacional bruto y formación de capital en España, 1849-1958: primer ensayo de estimación», en Martín Aceña, P., y Prados de la Escosura, L. (1985), pp. 17-51.

CECA (1975): Estadisticas básicas de España, 1900-1970, Madrid, CECA.

Comín Comín, F., y Martín Aceña, P. (1984): «La política monetaria y fiscal durante la Dictadura y la Segunda República», Papeles de Economia Española, núm. 20, pp. 236-261.

Crafts, N. F. R. (1983): «Gross National Product, 1870-1910: Some New Estimates», Explorations in Economic History, 20, pp. 387-401.

Fraile, P. (1985 a): «Crecimiento económico y demanda de acero», en Martín Aceña, P., y Prados de la Escosura, L. (1985), pp. 71-100.

- (1985 6): «El fracaso de la revolución industrial en España: un modelo cerrado de industrialización», Información Comercial Española, núm. 623, pp. 97-104.

Fuentes Quintana, E. (1975): Los principios de la imposición española y los problemas de su reforma, Madrid, Real Academia de Ciencias Morales y Políticas.

García Delgado, J. L. (1975): «Contribución al estudio del capitalismo español durante la dictadura de Primo de Riveraw, en Orígenes y desarrollo del capitalismo en España. Notas criticas, Madrid, Edicusa, pp. 139-215.

- (1980): *La política económica española de 1930: el "error Argüelles"», Papeles de Economia Española, núm. 1, pp. 43-47.

- (1983): «Autoritarismo político y tensiones económicas: un balance crítico de la política económica de la dictadura de Primo de Rivera en España (1923-1930) „, El Trimestre Económico, núm. 198, pp. 807-828.

García Delgado, J. L., y Roldán, S. (1974): La formación de la sociedad capitalista en España, 1914-1920, Madrid, CECA, 2 vols.

Garrabou, R., y SANZ, J. (1985): «La agricultura española durante el siglo xIx: cinmovilismo o cambio?», en Garrabou, R., y SAnz, J. (eds.): Historia agraria de la España contemporánea. 2. Expansión y crisis (1850-1900), Barcelona, Crítica, pp. 8-191.

González Portilla, M. (1981): La formación de la sociedad capitalista en el País Vasco (1876-1913), San Sebastián, L. Haranburu, 2 vols.

- (1985): «Las nuevas siderurgias vascas y los primeros sindicatos (carteles) siderúrgicosm, en Garcf́ Delgado, J. L. (comp.): La España de la Restauración política, económica, legislación y cultura, Madrid, Siglo XXI.

Instituto DE Estudios FISCALEs (1976): Datos básicos para la Historia Financiera de España, 1850-1975, Madrid, IEF, 2 vols.

- (1982): Cuentas del Estado español, 1924 a 1935, Madrid, Instituto de Estudios Fiscales.

INTERVEnCión General de la Administración del Estado (varios años): Liquidación Provisional del Presupuesto Extraordinario, Madrid, Imprenta y Encuadernación de la Suc. de M. Minuesa de los Ríos. 
Jiménez Araya, T. (1974): «Formación de capital y fluctuaciones económicas. Materiales para el estudio de un indicador: creación de sociedades mercantiles en España entre 1886 y 1970», Hacienda Pública Española, núm. 27, pp. 137-185.

Lindert, P. H., y Kindleberger, C. P. (1982): International Economics, Illinois, Richard D. Irwin Inc., pp. 137-138.

Martín ACEÑA, P. (1984): La politica monetaria en España, 1919.1935, Madrid, IEF, cap. 4.

- (1985): «Déficit público y política monetaria durante la Restauración», en MARTf́N ACEÑA, P., y PRADOS DE LA EscosurA, L. (1985), pp. 262-284.

Martín Áceña, P., y Prados de la Escosura, L. (1985): La nueva bistoria económica en España, Madrid, Tecnos.

Melguizo, A. (1979): „El presupuesto de Calvo Sotelow, Cuadernos Económicos de ICE, núm. 10, pp. 401-442, esp. 430-435.

NADAL, J. (1975): El fracaso de la revolución industrial en España, 1814-1913, Barcelona, Ariel.

Palafox, J. (1980): ala gran depresión de los años treinta y la crisis industrial españolaw, Investigaciones Económicas, núm. 11, pp. 4-46.

París Egullaz (1943): El movimiento de los precios en España. Su importancia para una politica de intervención, Madrid, CSIC.

PRAdos DE LA EscosurA, L. (1982): Comercio exterior y crecimiento económico en España, 1826-1913. Tendencias a largo plazo, Madrid, Servicio de Estudios del Banco de España.

- (1984): «El crecimiento económico moderno en España, 1830-1973: una comparación internacional», Papeles de Economia Española, núm. 20, pp. 151-154.

Sevilla, J. V. (1985): Economía política de la crisis española, Barcelona, Crítica.

Tortella, G. (1985): «Producción y productividad agraria, 1830-1930», en SÁNChezAlbornoz, N. (comp.): La modernización económica de España, 1830-1930, Madrid, Alianza, pp. 63-88.

Velarde, J. (1968): La politica econbmica de la Dictadura, Madrid, Guadiana. 